\title{
Stability and Tracking Properties in Predictive Control with Adaptation for Bilateral Teleoperation
}

\author{
Kouei Yoshida and Toru Namerikawa
}

\begin{abstract}
This paper deals with an environment estimation based predictive control for teleoperation with communication time delay. In this predictive control framework, the delayed position tracking and non-delayed force reflection are attempted. The goal of this paper is to propose a new control method which guarantees the stability, the convergence of position tracking error to zero and the small force tracking error theoretically. Proposed control structure is the $3 \mathrm{ch}$ architecture with environment estimation based force prediction. In this proposed method, the master controller implements force control with environmental force prediction while the slave controller implements position and force control. The theoretical analysis shows the stability, the convergence of position error to zero and the small force tracking error. Experimental results show the effectiveness of the proposed method.
\end{abstract}

\section{INTRODUCTION}

Teleoperation system can extend a human's reach to a remote site and has been developed and motivated by large variety of applications. It is well known that communication delay may destabilize the system and degrade the closed loop performance [1]. Hence it is necessary to improve the closed loop performance while preserving stability.

There are many control schemes proposed for dealing with the time delay in teleoperation systems [1], [2]. The passivity based approach developed in [3], [4] to guarantee stability by making the communication channel a passive transmission line. These schemes have been extended to provide position tracking performance improvement, stability for time varying delay and various other objectives [5][7]. These schemes [3]-[7] have been shown to be an efficient method for stabilizing a two-channel (2ch) architecture (involving transmission of only one quantity from master to the slave and vice versa). On the other hand, the four channel (4ch) architecture [8] (involving transmission of two quantities from master to the slave and vice versa) provide better performance than $2 \mathrm{ch}$ architecture under no communication delay [9]. This 4ch architecture have been extended to $3 \mathrm{ch}$ architecture in [10], [11] and the robustness of these architecture has been analyzed to deal with the time delay [10]. In [12], the wave theory [4] was incorporated in $4 \mathrm{ch}$ and $3 \mathrm{ch}$ architecture to stabilize the these architectures. These method [3]-[12] attempt delayed position tracking and force reflection while preserving stability but non-delayed position tracking and force reflection can not be achieved because they use delayed information without prediction.

K. Yoshida and T. Namerikawa are with Division of Electrical Engineering and Computer Engineering, Graduate School of Natural Science and Technology, Kanazawa University, Kakuma-machi, Kanazawa 9201192 JAPAN. yoshidalscl.ec.t.kanazawa-u.ac.jp and toruet.kanazawa-u.ac.jp
The predictive approaches have been developed in [13] [18]. [13]-[16] attempt the non-delayed position tracking or non-delayed force reflection or realization of delay-free dynamics. In these methods, the uncertainties of operator and environmental model cause prediction error and position tracking error and force tracking error. Practically, the dynamics of operator is very complicated and it is difficult to obtain accurate model. On the other hand, [17], [18] propose the environment estimation based predictive control for teleoperation with communication time delay. These controllers estimate the remote environment on-line and can deal with the uncertainty of remote environment In [17], the adaptive prediction was incorporated to the wave theory for reduction of the effect of delay. In [18], "pseudo" 2 ch channel architecture was proposed. In this method, the position tracking is delayed but non-delayed force reflection is attempted by Neural Network based force prediction. Furthermore, it is shown experimentally that the task is easier than conventional control method without prediction. However, the position tracking error and force tracking error performance and the stability are not analyzed theoretically. It is expected that the perfect position tracking is not achieved because they are similar to $2 \mathrm{ch}$ architecture. Finally, in [19], another method including environmental estimation was proposed but the stability analysis was not presented.

This paper deals with a environment estimation based predictive control for teleoperation with communication time delay. The goal of this paper is to propose a new control method which guarantee the stability, the convergence of position tracking error to zero and the small force tracking error theoretically. The proposed structure is the 3ch architecture with environment estimation based force prediction. The theoretical analysis show the stability, the convergence of position error to zero and the small force tracking error. Experimental results show the effectiveness of the proposed method.

\section{PRoblem Formulation}

\section{A. Modeling of Teleoperation System}

Assuming absence of friction and other disturbances and compensation of gravity effect, the master and slave robot dynamics with 1 degree of freedom are described as

$$
\begin{aligned}
M_{m} \ddot{x}_{m} & =F_{m}+F_{o p} \\
M_{s} \ddot{x}_{s} & =F_{s}-F_{e n v}
\end{aligned}
$$

where the subscripts " $m$ ", " $s$ " denote the master and the slave indexes respectively, $M_{m}, M_{s} \in \mathcal{R}$ are inertia of the 
robots, $x_{m}, x_{s} \in \mathcal{R}$ are the position of robots, $F_{m}, F_{s} \in \mathcal{R}$ are control inputs, $F_{o p}, F_{\text {env }} \in \mathcal{R}$ are the operator force and environmental force respectively. We assume that the operator force and environmental force satisfy following assumptions.

Assumption 1: The operator force $F_{o p}$ is bounded.

Assumption 2: slave contacts with following springdamper environment with constant parameter.

$$
\begin{aligned}
& F_{\text {env }}=B_{\text {env }} \dot{x}_{s}+K_{\text {env }}\left(x_{s}-x_{0}\right)=\boldsymbol{W}^{T} \boldsymbol{\theta}_{\text {env }} \\
& \boldsymbol{W}=\left[\begin{array}{llll}
x_{s} & \dot{x}_{s} & 1
\end{array}\right]^{T}, \boldsymbol{\theta}_{\text {env }}=\left[\begin{array}{lll}
K_{\text {env }} & B_{\text {env }} & -K_{\text {env }} x_{0}
\end{array}\right]^{T}
\end{aligned}
$$

where $K_{\text {env }}, B_{\text {env }}, x_{0} \in \mathcal{R}$ are stiffness, damping and equilibrium position respectively.

Remark 1: Practically, the remote environment may be time-varying nonlinear system. The control scheme in [18] can be applied for time-varying nonlinear environment by Neural Network based estimation. In our work, we focus on the stability and the position and force tracking performance in environment estimation based predictive control. Thus, only simple remote environment is treated and the extension of the class of environment is future work.

In this paper, environment parameter $\boldsymbol{\theta}_{\boldsymbol{e n} \boldsymbol{v}}$ is estimated online by least-squares estimator [17], [20], [21]. In the leastsquares estimater, it is necessary that $\boldsymbol{W}$ is persistently exciting for estimated parameter convergence to true value [20]. In our analysis, we assume that $\boldsymbol{W}$ is persistently exciting.

Assumption 3: $\boldsymbol{W}$ satisfies following infinite integral condition

$$
\lambda_{\min }\left\{\int_{0}^{t} \boldsymbol{W} \boldsymbol{W}^{T} d t^{\prime}\right\} \rightarrow \infty \text { as } t \rightarrow \infty .
$$

Remark 2: $\lambda_{\min }\left\{\int_{0}^{t} \boldsymbol{W} \boldsymbol{W}^{T} d t^{\prime}\right\}$ does not increase when the slave velocity is zero. However, it is expected that $\lambda_{\min }\left\{\int_{0}^{t} \boldsymbol{W} \boldsymbol{W}^{T} d t^{\prime}\right\}$ increase when the slave is moving from experimental results in section $\mathrm{V}$.

In this paper, the time varying delays are considered. It is assumed that the signal transmitted to the slave (master) from master (slave) is delayed as $T_{m}(t) \in \mathcal{R}\left(T_{s}(t) \in \mathcal{R}\right)$.

\section{B. Control Objective}

From a control theoretic point of view, the main goals of teleoperation are twofold, stability and transparency. Thus, we define the first control objective as follows

Control Objective 1: The system is stable in the sense of the boundedness of the position and velocity of master and slave.

The transparent system means that human operator feels as if directly interacting with the remote environment [22]. This is equivalent to the accurate position and force tracking $\left(x_{m}(t)=x_{s}(t), F_{o p}(t)=F_{e n v}(t)\right)$. However, in teleoperation system with communication time delay, it is difficult to achieve these accurate non-delayed position and force tracking bacause the informations of opposite side are delayed. Especially, non-delayed position tracking may require the prediction of the current time master position in slave side. This prediction is very difficult because the master is moved by the operator whose characteristics is very complicated and whose identification is very difficult. However, remote environmental force can be predicted and the non-delayed force reflection is possible if slave may contacts with identifiable remote environment.

In this paper, it is defined as control objective that the human operator feels as if directly interacting with the identfied virtual environment and the slave performs delayed position tracking and force tracking. This control objective is equivalent to achievement of following Control Objective 2,3 .

Control Objective 2: $x_{s}(t)-x_{m}\left(t-T_{m}(t)\right)=0$

Control Objective $3: F_{\text {env }}(t)-F_{o p}\left(t-T_{m}(t)\right)=0$

Control Objective 3 can be rewritten as $F_{\text {op }}(t)=F_{\text {env }}(t+$ $\left.T_{m}(t)\right)$. it means the non-delayed force reflection in the master side.

\section{Controller Design}

\section{A. Control Structure}

The proposed control structure is shown in Fig. 1. This structure is $3 \mathrm{ch}$ architecture [11] with environment estimation based force prediction in master side. In the slave side, the delayed informations of the master are used. On the other hand, environmental force is predicted based on estimated environment parameter $\hat{\boldsymbol{\theta}}_{\boldsymbol{e n \boldsymbol { v }}}\left(t-T_{s}(t)\right)$ in the master side.

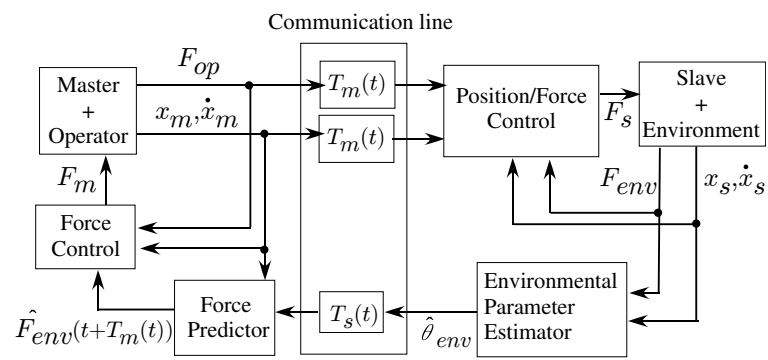

Fig. 1. Control structure

\section{B. Control Law}

To achieve the control objective, we propose the following control law with predicted force

$$
\begin{aligned}
F_{m}= & \left(K_{f}-1\right) F_{o p}-K_{f} \hat{F}_{e n v}\left(t+T_{m}(t)\right)-B \dot{x}_{m} \\
F_{s}= & F_{e n v}+\rho\left\{K_{p}\left(x_{m}\left(t-T_{m}(t)\right)-x_{s}\right)\right. \\
& +K_{d}\left(\dot{x}_{m}\left(t-T_{m}(t)\right)-\dot{x}_{s}\right) \\
& \left.\quad+K_{f}\left(F_{o p}\left(t-T_{m}(t)\right)-F_{e n v}\right)-B \dot{x}_{s}\right\} .
\end{aligned}
$$

where, $K_{f}, K_{p}, K_{d}, B \in \mathcal{R}$ are design parameter respectively, $\rho$ is defined as $\rho=M_{s} / M_{m}$ from consideration of the results in [23]. $\hat{F}_{\text {env }}\left(t+T_{m}(t)\right)$ is predicted value of environmental force at time $t+T_{m}(t)$. This is calculated by following equation

$$
\begin{aligned}
\hat{F}_{\text {env }}\left(t+T_{m}(t)\right)= & \hat{B}_{\text {env }}\left(t-T_{s}(t)\right) \dot{x}_{m}(t) \\
& +\hat{K}_{\text {env }}\left(t-T_{s}(t)\right) x_{m}(t)+\hat{C}\left(t-T_{s}(t)\right) .
\end{aligned}
$$


where $\hat{B}_{e n v}, \hat{K}_{e n v}, \hat{C}$ are estimated parameter of environment parameter $B_{e n v}, K_{e n v},-K_{e n v} x_{0}$ which is estimated by following least-squares estimator

$$
\begin{aligned}
\dot{\hat{\boldsymbol{\theta}}}_{\text {env }} & =-\boldsymbol{P} \boldsymbol{W}\left\{\boldsymbol{W}^{T} \hat{\boldsymbol{\theta}}_{\text {env }}-F_{\text {env }}\right\} \\
\frac{d}{d t} \boldsymbol{P}^{-1} & =\boldsymbol{W} \boldsymbol{W}^{T}, \boldsymbol{P}(0)=p_{0} \boldsymbol{I} \\
\left(\hat{\boldsymbol{\theta}}_{\text {env }}\right. & \left.=\left[\begin{array}{lll}
\hat{K}_{\text {env }} & \hat{B}_{\text {env }} & \hat{C}
\end{array}\right]^{T}\right) .
\end{aligned}
$$

where $p_{0}$ is initial value of the matrix $P(t)$. Combining the control law (7)(8) with dynamics of robots (1)(2) yields following closed loop system

$$
\begin{aligned}
M_{m} \ddot{x}_{m}= & -\left(K_{f} B_{e n v}+B\right) \dot{x}_{m}-K_{f} K_{e n v} x_{m} \\
& -K_{f} C+K_{f} F_{o p} \\
& -K_{f} \tilde{B}_{e n v} \dot{x}_{m}-K_{f} \tilde{K}_{e n v} x_{m}-K_{f} \tilde{C} \\
M_{m} \ddot{x}_{s}= & -B \dot{x}_{s}+K_{p}\left(x_{m}\left(t-T_{m}(t)\right)-x_{s}\right) \\
& +K_{d}\left(\dot{x}_{m}\left(t-T_{m}(t)\right)-\dot{x}_{s}\right) \\
& +K_{f}\left(F_{o p}\left(t-T_{m}(t)\right)-F_{\text {env }}\right)
\end{aligned}
$$

where $\tilde{K}_{\text {env }}, \tilde{B}_{\text {env }}, \tilde{C}$ are the estimation errors of environment parameter which are defined as follows

$$
\begin{aligned}
\tilde{K}_{e n v} & =\hat{K}_{e n v}\left(t-T_{s}(t)\right)-K_{e n v} \\
\tilde{B}_{e n v} & =\hat{B}_{e n v}\left(t-T_{s}(t)\right)-B_{e n v} \\
\tilde{C} & =\hat{C}\left(t-T_{s}(t)\right)+K_{e n v} x_{0} .
\end{aligned}
$$

In the next section, the closed loop stability and performance are analyzed based on the closed loop system dynamics $(10)(11)(13)(14)$.

\section{Stability and Performance Analysis}

In this section, we analyze the boundedness of position and velocity, convergence of position error between the master and slave and force tracking performance.

To facilitate the analysis in this section, we describe the following lemma for convergence of environment parameter estimation.

Lemma 1: [20] Consider the environment estimation described by (10)(11). Then, the parameter estimation error $\tilde{\boldsymbol{\theta}}_{\text {env }}=\hat{\boldsymbol{\theta}}_{\boldsymbol{e n \boldsymbol { v }}}(t)-\boldsymbol{\theta}_{\boldsymbol{e n \boldsymbol { v }}}$ satisfy following equation.

$$
\left\|\tilde{\boldsymbol{\theta}}_{\text {env }}\right\| \leq\left\{\lambda_{\min }\left(\boldsymbol{I}+p_{0} \int_{0}^{t} \boldsymbol{W} \boldsymbol{W}^{T} d t^{\prime}\right)\right\}^{-\frac{1}{2}}\left\|\tilde{\boldsymbol{\theta}}_{\text {env }}(0)\right\|
$$

Thus, the parameter estimation errors are bounded. Furthermore, if Assumption 3 is satisfied, the parameter estimation errors converge to true value as follows

$$
\left\|\tilde{\boldsymbol{\theta}}_{\text {env }}\right\| \rightarrow 0 \text { as } t \rightarrow \infty .
$$

Using this lemma, we can prove the boundedness of position and velocity of the master as following lemma.

Lemma 2: (Boundedness of the master state) Consider the dynamics of the master (13) and parameter update law (10)(11) with assumption 1-3. Then, the position and velocity of the master $x_{m}, \dot{x}_{m}$ are bounded.

Proof: At first, we introduce the state space representations of (13) as follows

$$
\dot{\boldsymbol{X}}_{m}=\left(\boldsymbol{A}_{m}+\tilde{\boldsymbol{A}}_{m}\right) \boldsymbol{X}_{m}+\boldsymbol{d}
$$

$$
\begin{aligned}
\boldsymbol{X}_{m} & =\left[\begin{array}{l}
x_{m} \\
\dot{x}_{m}
\end{array}\right], \boldsymbol{d}=\left[\begin{array}{c}
0 \\
M_{m}^{-1}\left\{K_{f} F_{o p}-K_{f} \hat{C}\left(t-T_{s}(t)\right)\right\}
\end{array}\right], \\
\boldsymbol{A}_{m} & =\left[\begin{array}{cc}
0 & 1 \\
-M_{m}^{-1} K_{f} K_{e n v} & -M_{m}^{-1}\left(K_{f} B_{e n v}+B\right)
\end{array}\right] \\
\tilde{\boldsymbol{A}}_{m} & =\left[\begin{array}{cc}
0 & 0 \\
-M_{m}^{-1} K_{f} \tilde{K}_{e n v} & -M_{m}^{-1} K_{f} \tilde{B}_{e n v}
\end{array}\right] .
\end{aligned}
$$

If $K_{e n v}>0, \boldsymbol{A}_{\boldsymbol{m}}$ is stable matrix. $\hat{C}, F_{o p}$ are bounded from Lemma 1,2 respectively. Therefore, $\boldsymbol{d}$ is bounded and there exist the constant $\epsilon_{d}$ satisfying following inequality

$$
\|\boldsymbol{d}\| \leq \epsilon_{d}
$$

The elements of $\tilde{A}_{m}$ are bounded and converge to zero from Lemma 1. Thus, there exist the variable $\alpha(t)$ satisfying following equations.

$$
\tilde{\boldsymbol{A}}_{\boldsymbol{m}}^{\boldsymbol{T}}(t) \tilde{\boldsymbol{A}}_{\boldsymbol{m}}(t) \leq \alpha(t) \boldsymbol{I}, \alpha(t) \rightarrow 0 \text { as } t \rightarrow \infty
$$

If $\boldsymbol{A}_{\boldsymbol{m}}$ is stable, there exist constants $\boldsymbol{P}_{\mathbf{1}}>0, \gamma_{1}>0, \gamma_{2}>0$ satisfying following inequality

$$
\boldsymbol{A}_{m} \boldsymbol{P}_{\mathbf{1}}+\boldsymbol{P}_{\mathbf{1}} \boldsymbol{A}_{m}+\gamma_{1}^{-1} \boldsymbol{P}_{\mathbf{1}} \boldsymbol{P}_{\mathbf{1}}+\gamma_{2}^{-1} \boldsymbol{P}_{\mathbf{1}} \boldsymbol{P}_{\mathbf{1}}+\boldsymbol{I}<0 .
$$

We consider the lyapunov function candidate $V_{1}=$ $\boldsymbol{X}_{m}^{T} \boldsymbol{P}_{1} \boldsymbol{X}_{m}$ by using the solution $\boldsymbol{P}_{\mathbf{1}}$ of (21). The derivative of $V_{1}$ along the solution trajectories of the master dynamics gives

$$
\begin{aligned}
\dot{V}_{1}= & \boldsymbol{X}_{m}^{T}\left\{\boldsymbol{A}_{m}^{T} \boldsymbol{P}_{1}+\boldsymbol{P}_{1} \boldsymbol{A}_{m}\right\} \boldsymbol{X}_{m} \\
& +\boldsymbol{X}_{m}^{T} \tilde{A}_{m}^{T} \boldsymbol{P}_{1} \boldsymbol{X}_{m}+\boldsymbol{X}_{m}^{T} \boldsymbol{P}_{1} \tilde{A}_{m} \boldsymbol{X}_{m} \\
& +\boldsymbol{d}^{T} \boldsymbol{P}_{\mathbf{1}} \boldsymbol{X}_{m}+\boldsymbol{X}_{m}^{T} \boldsymbol{P}_{1} d .
\end{aligned}
$$

Using the property that $\boldsymbol{a}^{T} \boldsymbol{b}+\boldsymbol{b}^{T} \boldsymbol{a} \leq \gamma \boldsymbol{a}^{T} \boldsymbol{a}+\gamma^{-1} \boldsymbol{b}^{T} \boldsymbol{b},(\gamma>$ $0)$, we have

$$
\begin{aligned}
\dot{V}_{1} \leq & \boldsymbol{X}_{m}^{T}\left\{\boldsymbol{A}_{m} \boldsymbol{P}_{\mathbf{1}}+\boldsymbol{P}_{\mathbf{1}} \boldsymbol{A}_{m}+\gamma_{1}^{-1} \boldsymbol{P}_{\mathbf{1}} \boldsymbol{P}_{\mathbf{1}}\right. \\
& \left.+\gamma_{2}^{-1} \boldsymbol{P}_{\mathbf{1}} \boldsymbol{P}_{\mathbf{1}}+\boldsymbol{I}\right\} \boldsymbol{X}_{m}+\gamma_{1} \boldsymbol{X}_{m}^{T} \tilde{\boldsymbol{A}}_{m}^{T} \tilde{\boldsymbol{A}}_{m} \boldsymbol{X}_{m} \\
& +\gamma_{2} \boldsymbol{d}^{T} \boldsymbol{d}-\boldsymbol{X}_{m}^{\boldsymbol{T}} \boldsymbol{X}_{m} .
\end{aligned}
$$

Using the inequality (21), we have

$$
\dot{V}_{1} \leq \gamma_{1} \boldsymbol{X}_{m}^{\boldsymbol{T}} \tilde{A}_{m}^{T} \tilde{A}_{m} \boldsymbol{X}_{m}+\gamma_{2} \boldsymbol{d}^{T} \boldsymbol{d}-\boldsymbol{X}_{m}^{\boldsymbol{T}} \boldsymbol{X}_{m} .
$$

Consideration of (19), (20) gives

$$
\dot{V}_{1} \leq-\left(1-\gamma_{1} \alpha(t)\right)\left\|\boldsymbol{X}_{\boldsymbol{m}}\right\|^{2}+\gamma_{2} \epsilon_{d}^{2}
$$

where $\alpha(t)$ is maximum eigen value of $\tilde{\boldsymbol{A}}_{m}^{T} \tilde{\boldsymbol{A}}_{m}$ satisfying following equations

$$
\begin{aligned}
\alpha(t) & =\left(\frac{K_{f}}{M_{m}}\right)^{2}\left\{\tilde{K}_{e n v}^{2}+\tilde{B}_{e n v}^{2}\right\} \\
& \leq\left(\frac{K_{f}}{M_{m}}\right)^{2}\left\|\tilde{\boldsymbol{\theta}}_{\text {env }}\left(t-T_{s}\right)\right\|^{2} .
\end{aligned}
$$

$\left\|\tilde{\boldsymbol{\theta}}_{\text {env }}\left(t-T_{s}\right)\right\|$ decreases as Lemma 1. Thus, there exist finite time $T_{0}$ satisfying following equation for any constant $1>\epsilon_{1}>0$

$$
\left(1-\gamma_{1} \alpha(t)\right) \geq \epsilon_{1}>0, \forall t \geq T_{0} .
$$

Furthermore, from the boundedness of $\gamma_{1} \alpha(t)$, there exist constant $\epsilon_{2}>0$ satisfying following equation

$$
\left(1-\gamma_{1} \alpha(t)\right) \geq-\epsilon_{2}>-\infty, \forall t<T_{0} .
$$


From these equations (26)(27), we have

$$
\begin{aligned}
& \dot{V}_{1} \leq \epsilon_{2}\left\|\boldsymbol{X}_{\boldsymbol{m}}\right\|^{2}+\gamma_{2} \epsilon_{d}^{2}, \forall t<T_{0} \\
& \dot{V}_{1} \leq-\epsilon_{1}\left\|\boldsymbol{X}_{\boldsymbol{m}}\right\|^{2}+\gamma_{2} \epsilon_{d}^{2}, \forall t \geq T_{0} .
\end{aligned}
$$

Using the definition of $V_{1}$, the following differential inequalities are obtained

$$
\begin{aligned}
& \dot{V}_{1} \leq \frac{\epsilon_{2}}{\lambda_{\min }\left(\boldsymbol{P}_{\mathbf{1}}\right)} V_{1}+\gamma_{2} \epsilon_{d}^{2}, \forall t<T_{0} \\
& \dot{V}_{1} \leq \frac{-\epsilon_{1}}{\lambda_{\max }\left(\boldsymbol{P}_{\mathbf{1}}\right)} V_{1}+\gamma_{2} \epsilon_{d}^{2}, \forall t \geq T_{0} .
\end{aligned}
$$

The solution of this differential inequalities are given as follows

$$
\begin{aligned}
V_{1}(t) \leq & V_{1}(0) \boldsymbol{e}^{\frac{\epsilon_{2} t}{\lambda_{\min }\left(\boldsymbol{P}_{\mathbf{1}}\right)}} \\
& +\frac{\gamma_{2} \epsilon_{d}^{2} \lambda_{\min }\left(\boldsymbol{P}_{\mathbf{1}}\right)}{\epsilon_{2}}\left(1-e^{\frac{\epsilon_{2} t}{\lambda_{\min }\left(\boldsymbol{P}_{\mathbf{1}}\right)}}\right), \forall t<T_{0} \\
V_{1}(t) \leq & V_{1}\left(T_{0}\right) e^{\frac{-\epsilon_{1}\left(t-T_{0}\right)}{\lambda_{\max }\left(\boldsymbol{P}_{1}\right)}} \\
& +\frac{\gamma_{2} \epsilon_{d}^{2} \lambda_{\max }\left(\boldsymbol{P}_{\mathbf{1}}\right)}{\epsilon_{1}}\left(1-e^{\frac{-\epsilon_{1}\left(t-T_{0}\right)}{\lambda_{\max }\left(\boldsymbol{P}_{\mathbf{1}}\right)}}\right), \forall t \geq T_{0}
\end{aligned}
$$

From these solutions and boundedness of $T_{0}, V_{1}$ is bounded. The boundedness of $V_{1}$ imply the boundedness of $\boldsymbol{X}_{\boldsymbol{m}}$. Next, we consider the position error between master and slave. The position error converges to zero as following lemma.

Lemma 3: (Position tracking) Consider the closed loop system (10)(11) (13)(14) with Assumptions 1-3. Then the position error $e_{p}=x_{m}-x_{s}\left(t+T_{m}(t)\right)$ and velocity error $\dot{e}_{p}=\dot{x}_{m}-\dot{x}_{s}\left(t+T_{m}(t)\right)$ converge to zero.

Proof: By shifting the time $T_{m}(t)[s]$, the slave dynamics (14) is rewritten as follows

$$
\begin{aligned}
M_{m} \ddot{x}_{s}\left(t+T_{m}(t)\right)= & \left.-B \dot{x}_{s}\left(t+T_{m}(t)\right)\right) \\
& +K_{p}\left(x_{m}-x_{s}\left(t+T_{m}(t)\right)\right) \\
& +K_{d}\left(\dot{x}_{m}-\dot{x}_{s}\left(t+T_{m}(t)\right)\right) \\
& +K_{f}\left(F_{o p}(t)-F_{\text {env }}\left(t+T_{m}(t)\right)\right) .
\end{aligned}
$$

Using the equation (3) of $F_{\text {env }}$, we have

$$
\begin{aligned}
M_{m} \ddot{x}_{s}\left(t+T_{m}(t)\right)= & K_{p}\left(x_{m}-x_{s}\left(t+T_{m}(t)\right)\right) \\
& +K_{d}\left(\dot{x}_{m}-\dot{x}_{s}\left(t+T_{m}(t)\right)\right)+K_{f} F_{o p} \\
& -\left(K_{f} B_{e n v}+B\right) \dot{x}_{s}\left(t+T_{m}(t)\right) \\
& -K_{f} K_{e n v} x_{s}\left(t+T_{m}(t)\right)+K_{f} K_{\text {env }} x_{0} .
\end{aligned}
$$

Substracting (35) from (13), we have following error system.

$$
\begin{aligned}
& M_{m} \ddot{e}_{p}+\left\{K_{d}+K_{f} B_{e n v}+B\right\} \dot{e}_{p}+\left\{K_{p}+K_{f} K_{e n v}\right\} e_{p}=d_{p} \\
& \left(d_{p}=-K_{f} \tilde{B}_{e n v} \dot{x}_{m}-K_{f} \tilde{K}_{e n v} x_{m}+K_{f} \tilde{C}\right) .
\end{aligned}
$$

If Assumptions 1-3 are satisfied, parameter estimation error $\tilde{B}_{\text {env }}, \tilde{K}_{\text {env }}, \tilde{C}$ converge to zero from Lemma 1. Furthermore, the master position and velocity $x_{m}, \dot{x}_{m}$ are bounded from lemma 2. Therefore, the variable $d_{p}$ consist of the terms which converge to zero as follows

$$
d_{p}=-K_{f} \underbrace{\tilde{B}_{\text {env }}}_{\rightarrow 0} \underbrace{\dot{x}_{m}}_{\text {bounded }}-K_{f} \underbrace{\tilde{K}_{\text {env }}}_{\rightarrow 0} \underbrace{x_{m}}_{\text {bounded }}+K_{f} \underbrace{\tilde{C}}_{\rightarrow 0} .
$$

Thus, we have $\lim _{t \rightarrow \infty} d_{p} \rightarrow 0$. By defining input as $d_{p}$, and output as $\left[\begin{array}{ll}e_{p} & \dot{e}_{p}\end{array}\right]^{T}$, the transfer function $G(s)$ is given as follows

$$
G(s)=\left[\begin{array}{c}
\frac{M_{m}^{-1}}{s^{2}+M_{m}^{-1}\left\{K_{d}+K_{f} B_{e n v}+B\right\} s+M_{m}^{-1}\left\{K_{p}+K_{f} K_{e n v}\right\}} \\
\frac{M_{m}^{-1} s}{s^{2}+M_{m}^{-1}\left\{K_{d}+K_{f} B_{e n v}+B\right\} s+M_{m}^{-1}\left\{K_{p}+K_{f} K_{e n v}\right\}}
\end{array}\right] .
$$

This transfer function is stable. From the stability of the transfer function and the fact of $\lim _{t \rightarrow \infty} d_{p} \rightarrow 0$, we can prove $\lim _{t \rightarrow \infty} e_{p}, \dot{e}_{p} \rightarrow 0$ by using the input-output stability of linear system [24].

The next lemma shows the force tracking performance.

Lemma 4: (Force tracking) Consider the closed loop system (10)(11) (13)(14) with Assumptions 1-3. Then, the force error $F_{o p}-F_{\text {env }}\left(t+T_{m}(t)\right)$ satisfy following equation.

$$
\begin{aligned}
& F_{o p}-F_{e n v}\left(t+T_{m}(t)\right) \\
=\quad & \left(\hat{F}_{e n v}\left(t+T_{m}(t)\right)-F_{e n v}\left(t+T_{m}(t)\right)\right) \\
& +K_{f}^{-1} M_{m} \ddot{x}_{m}+K_{f}^{-1} B \dot{x}_{m}
\end{aligned}
$$

Furthermore, the term of force prediction error $\left(\hat{F}_{\text {env }}(t+\right.$ $\left.\left.T_{m}(t)\right)-F_{\text {env }}\left(t+T_{m}(t)\right)\right)$ converge to zero in steady state.

Proof: (38) can be obtained by transformation of master dynamics. The force prediction error term consist of the terms which converge to zero.

$$
\begin{aligned}
& \hat{F}_{\text {env }}\left(t+T_{m}(t)\right)-F_{\text {env }}\left(t+T_{m}(t)\right) \\
& =B_{\text {env }} \underbrace{\dot{e}_{p}}_{\rightarrow 0}+K_{\text {env }} \underbrace{e_{p}}_{\rightarrow 0}+\underbrace{\tilde{B}_{\text {env }}}_{\rightarrow 0} \underbrace{\dot{x}_{m}(t)}_{\text {bounded }} \\
& +\underbrace{\tilde{K}_{\text {env }}}_{\rightarrow 0} \underbrace{x_{m}(t)}_{\text {bounded }}+\underbrace{\tilde{C}}_{\rightarrow 0}
\end{aligned}
$$

where we use the convergence of $\tilde{K}_{e n v}, \tilde{B}_{e n v}, \tilde{C}$ from Lemma 1, boundeness of $x_{m}, \dot{x}_{m}$ from Lemma 2 and the convergence of $e_{p}, \dot{e_{p}}$ from Lemma 3. Therefore, $\lim _{t \rightarrow \infty} \hat{F}_{\text {env }}\left(t+T_{m}(t)\right)-F_{\text {env }}\left(t+T_{m}(t)\right)=0$.

From these Lemmas 1-4, the achievement of control objectives are concluded as the following theorem.

Theorem 1: Consider the closed loop system (10)(11) (13)(14) with Assumptions 1-3. Then, the following three facts are given.

1) The position and velocity of master and slave are bounded. Thus, the system is stable, i.e. Control $\mathrm{Ob}$ jective 1 is achieved.

2) The position error and velocity errors between the master and slave converge to zero in steady state. Thus, Control Objective 2 is achieved.

3) The force error in steady state is given as follows $F_{o p}-F_{\text {env }}\left(t+T_{m}\right)=K_{f}^{-1} M_{m} \ddot{x}_{m}+K_{f}^{-1} B \dot{x}_{m}$

Thus, Control Objective 3 is achieved if $\ddot{x}_{m}, \dot{x}_{m}=0$. Furthermore, if $K_{f}$ is large or $M_{m}, B$ are small, force tracking error is small even if $\ddot{x}_{m}, \dot{x}_{m} \neq 0$.

Proof: This is clear from Lemmas 1-4.

Remark 3: The above theorem is also given if the following LTI operator model [10], [16], [22] is used.

$$
F_{o p}=-M_{o p} \ddot{x}_{m}-B_{o p} \dot{x}_{m}-K_{o p} x_{m}+F_{o p}^{*}
$$

where, $M_{o p}, B_{o p}, K_{o p} \in \mathcal{R}$ are operator mass, damping and stiffness and $F_{o p}^{*} \in \mathcal{R}$ is external force. If slave do not contact any environment $\left(K_{\text {env }}=0, B_{\text {env }}=0\right), \boldsymbol{A}_{\boldsymbol{m}}$ is not 
stable matrix. In this case, the boundedness of master state can not be proven by using only Assumption 1 for operator force. However, if the model (39) is used for the analysis, the boundedness of master state can be proven even if $K_{e n v}=$ $0, B_{e n v}=0$.

\section{EXPERIMENTAL EVALUATION}

In this section, the stability and the performance of the position and force tracking are evaluated experimentally. The experiment was carried out on a pair of identical 2 degree of freedom robots as shown in Fig. 2. We use DS1104 (dSPACE Inc.) as a real-time calculating machine and $1[\mathrm{~ms}]$ sampling rate is obtained. Experiment has been done with an artificial time delay $T_{m}=T_{s}=1[\mathrm{~s}]$. We use the environment of spring as shown in Fig. 3. Operator and environmental forces were measured by force sensor. In this paper, we discussed for only 1 degree of freedom linear robot but the robots used in experiment are multi degree of freedom nonlinear system. Thus, we use the linearization by adaptive impedance control [25] as [14], [16] and the controllers are designed for $x, y$ independently. The detail of the controller is shown in appendix.

Experimental results are shown in Figs. 4-10. We show only data of $y$ direction here. Fig. 4 shows the estimation of environment parameters. As shown in Fig. 4, the estimated parameters converge to certain value after 12 [s]. Fig. 5 shows $\lambda_{\min }\left\{\int_{0}^{t} \boldsymbol{W}_{\boldsymbol{y}} \boldsymbol{W}_{\boldsymbol{y}}^{\boldsymbol{T}} d t^{\prime}\right\}$ (the definition of $\boldsymbol{W}_{\boldsymbol{y}}$ is shown in appendix). This figure shows the increase of $\lambda_{\min }\left\{\int_{0}^{t} \boldsymbol{W}_{\boldsymbol{y}} \boldsymbol{W}_{\boldsymbol{y}}^{\boldsymbol{T}} d t^{\prime}\right\}$. Thus, it is expected that the Assumption 3 is satisfied. Fig. 6 shows the master and slave position and Fig. 8 shows the operator and environmental force. Figs. 7, 9 are modification of Figs. 6, 8 for comparison where the master robot responses are shifted to $T_{m}$ [s] to cancel the communication delay Figs. 6-9 show small position and force error after 12 [s] due to convergence of estimated parameter. The position and force errors in steady state may be attributed to the friction disturbance and unmodelled dynamics of robots and environment. Finally, Fig. 10 is comparison between predicted environment force and true value. This figure show small prediction error after 12[s]. In this case, initial parameter estimation error is large (initial values of the

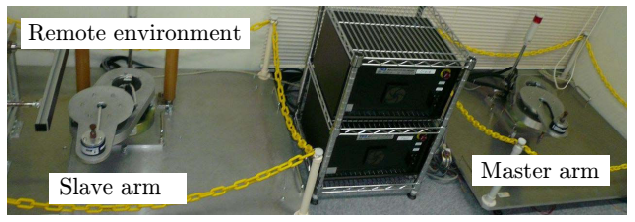

Fig. 2. Experimental setup

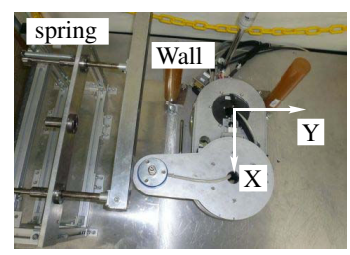

Fig. 3. Remote environment parameter estimation are zero). If initial parameter estimation error is small, the prediction error may converge quickly. The convergence of prediction error is also depend on time delay. The delay of the estimated parameter from slave to master cause the delay of the convergence of prediction error.

\section{CONCLUSION}

In this paper, we proposed new control method in environment estimation based predictive control. The proposed structure was the $3 \mathrm{ch}$ architecture with environment estimation based force prediction. The theoretical analysis showed the stability, the convergence of position error to zero and the small force tracking error. Experimental results showed the effectiveness of the proposed method. The future work is the extension of the class of environment.
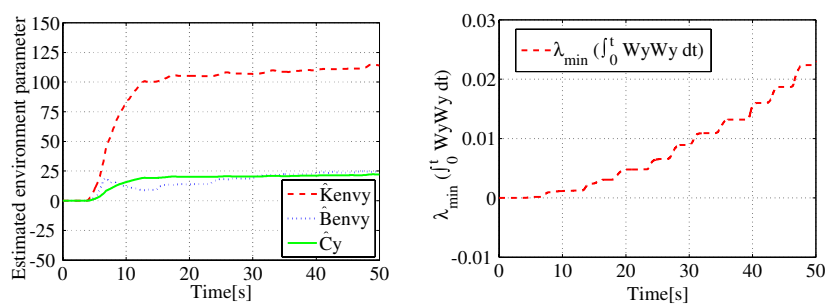

Fig. 4. Estimation of environment Fig. 5. Confirmation of integral parameter condition

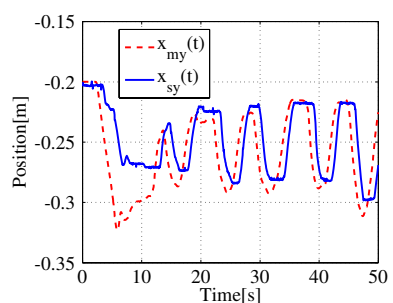

Fig. 6. Master and slave position

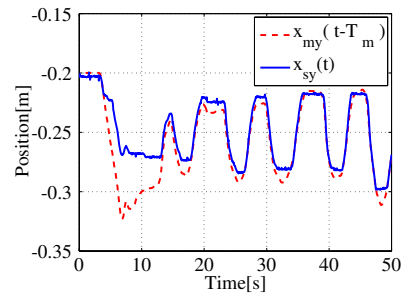

Fig. 7. Master and slave position (shifted)
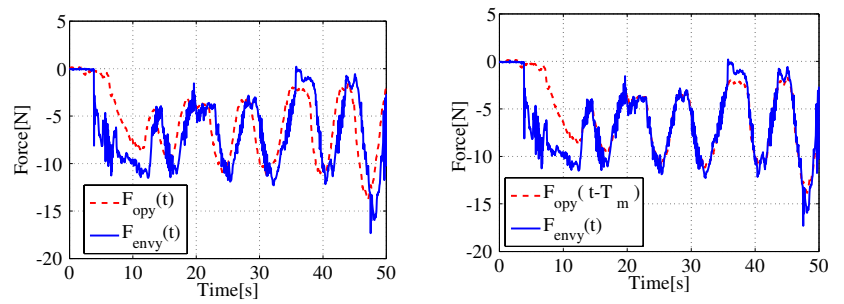

Fig. 8. Operator and environmental Fig. 9. Operator and environmental force force (shifted)

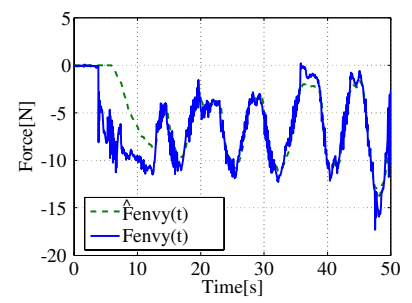

Fig. 10. Predicted and true values of environmental force 


\section{REFERENCES}

[1] P. F. Hokayem and M. W. Spong, "Bilateral teleoperation: An historical survey," Automatica, vol. 42, no. 12, pp. 2035-2057, 2006.

[2] P. Arcara and C. Melchiorri, "Control schemes for teleoperation with time delay: A comparative study," Robt. Auton. Syst., no. 38, pp. 49-64, 2002.

[3] R. J. Anderson and M. W. Spong, "Bilateral control of teleoperators with time Delay," IEEE Trans. Automat. Contr., vol. 34, no. 5, pp. 494-501, 1989.

[4] G. Niemeyer and J. J. E. Slotine, "Stable adaptive teleoperation," IEEE J. Oceanic Eng., vol. 16, no. 1, pp. 152-162, 1991.

[5] N. Chopra and M. W. Spong, "Adaptive synchronization of bilateral teleoperators with time delay," Advances in Telerobotics, springer, pp. 257-270, 2007.

[6] N. Chopra, M. W. Spong, S. Hirche and M. Buss, "Bilateral teleoperation over the internet,"in Proc. Amer. Contr. Conf., pp. 155-160, 2003.

[7] H. Kawada and T. Namerikawa, "Bilateral Control of Nonlinear Teleoperation with Time Varying Communication Delays," in Proc. Amer. Contr. Conf., pp. 189-194, 2008.

[8] D A. Lawrence, "Stability and transparency in bilateral teleoperation," IEEE Trans. Robot. Autom., vol.9, no.5, pp. 624-637, 1993.

[9] I. Aliaga, A. Rubio and E. Sanchez, "Experimental quantitative comparison of different control architectures for master-slave teleoperation," IEEE Trans. Contr. Syst. Technol., vol.12, no.1, pp. 2-11, 2004.

[10] K. Hashtrudi-Zaad, S. E. Salcudean, "Transparency in time-delayd systems and the effect of local force feedback for transparent teleoperation," IEEE Trans. Robot. Autom., vol.18, no.1, pp. 108-114, 2002.

[11] R. Kubo, N. Iiyama, K. Natori, K. Ohnishi, H. Furukawa, "Performance analysis of a three-channel control architecture for bilateral teleoperation with time delay," IEEJ Trans. IA, vol. 127, no.12, pp. 1224-1230, 2007.

[12] A. Aziminejad, M. Tavakoli, R. V. Patel and M. Moallem, "Transparent time-delayed bilateral teleoperation using wave variables," IEEE Trans. Contr. Syst. Technol., vol. 16, no. 3, pp. 548-555, 2008.

[13] Y. J. Pan, C. Canudas-de-Wit and O. Sename, "A new predictive approach for bilateral teleoperation with applications to drive-by-wire systems," IEEE Trans. Robot. , vol.10, no.6, pp. 1146-1162, 2006.

[14] K. Yoshida, T. Namerikawa, "Predictive PD Control for Teleoperation with Communication Time Delay," in Proc. 17th IFAC World Congr., pp. 12703-12708, 2008.

[15] K. Yoshida, T. Namerikawa and O. Sawodny, "A State Predictor for Bilateral Teleoperation with Communication Time Delay," in Proc. 47th IEEE Conf. Dec. Contr., pp. 4590-4595, 2008.

[16] S. Sirouspour, A. Shahdi, "Model predictive control for transparent teleoperation under communication time delay," IEEE Trans. Robot., vol.22, no.6, pp. 1131-1145, 2006.

[17] H. Ching , W.J. Book, "Internet-based bilateral teleoperation based on wave variable with semi-adaptive predictor and direct drift control" J Dyn. Syst. Measure. Contr., vol. 128, pp. 86-93, 2006.

[18] A. C. Smith and K. Hashtrudi-Zadd, "Adaptive teleoperation using neural network-based predictive control," in Proc. Conf. Contr. Appl., pp. 1269-1274, 2005.

[19] K. Hashtrudi-Zaad and S. E. Salcudean, "Adaptive transparent impedance reflecting teleoperation," in Proc. IEEE Int. Conf. Robot. Autom., vol. 2, pp. 1369-1374, 1996.

[20] J. J. E. Slotine and W. Li, Applied nonlinear control, PrenticeHall, 1991.

[21] L. Biagotti and C. Melchiorri, "Environment estimation in teleoperation systems," Advances in Telerobotics, springer, pp. 212-230, 2007.

[22] Y. Yokokohji and T. Yoshikawa, "Bilateral control of master-slave manipulators for ideal kinesthetic coupling - formulation and experiment," IEEE Trans. Robot. Autom., vol.10, no.5, pp 605-619, 1994.

[23] J. M. Azorin, R. Aracil, J. M. Sabater, C. Perez and N. M. Garcia, "Transparent bilateral control architecture by state convergence for teleorobotics," in Proc. 17th IFAC World Congr., pp. 12721-12726, 2008.

[24] H. L. Trentelman, A.A. Stoorvogel and M.L.J. Hautus, "Control theory for linear systems," Springer, 2000.

[25] W. S. Lu and Q. H. Meng, "Impedance control with adaptation," IEEE Trans. Robot. Autom., vol.7, no.3, pp. 408-415, 1991.

\section{APPENDIX}

\section{A. Controller for Experimental Evaluation}

In the experiment, we use the linearization by adaptive impendace control [14], [16], [25]. This appendix show the detail of this controller. The dynamics of the robots in experiment are described as

$$
\begin{array}{r}
M_{m}\left(\boldsymbol{q}_{m}\right) \ddot{\boldsymbol{q}}_{m}+\boldsymbol{C}_{m}\left(\boldsymbol{q}_{m}, \dot{\boldsymbol{q}}_{m}\right) \dot{\boldsymbol{q}}_{m}=\tau_{m}+\boldsymbol{J}_{m}^{T} \boldsymbol{F}_{\text {op }} \\
\boldsymbol{M}_{\boldsymbol{s}}\left(\boldsymbol{q}_{s}\right) \ddot{\boldsymbol{q}}_{s}+\boldsymbol{C}_{\boldsymbol{s}}\left(\boldsymbol{q}_{s}, \dot{\boldsymbol{q}}_{s}\right) \dot{\boldsymbol{q}}_{s}=\tau_{s}-\boldsymbol{J}_{s}^{T} \boldsymbol{F}_{\text {env }}
\end{array}
$$

The subscript $m, s$ denote the master and the slave indexes, and we define $i$ as $i=m, s . \boldsymbol{q}_{\boldsymbol{i}} \in \mathcal{R}^{2}$ is joint anlgle, $M_{\boldsymbol{i}} \in \mathcal{R}^{2 \times 2}$ are inertia matrices, $\boldsymbol{C}_{\boldsymbol{i}} \boldsymbol{q}_{\boldsymbol{i}} \in \mathcal{R}^{2}$ are centrifugal and Coriolis torque vectors. $\boldsymbol{J}_{\boldsymbol{i}} \in \mathcal{R}^{2 \times 2}$ are Jacobian. $\boldsymbol{\tau}_{\boldsymbol{m}}, \boldsymbol{\tau}_{\boldsymbol{s}} \in \mathcal{R}^{2}$ are input torque. $\boldsymbol{F}_{\boldsymbol{o p}}, \boldsymbol{F}_{\boldsymbol{e n} \boldsymbol{v}} \in \mathcal{R}^{2}$ are operator and environmental force. Using linearity in the parameters, (40)(41) are rewritten as $\boldsymbol{M}_{\boldsymbol{i}}\left(\boldsymbol{q}_{\boldsymbol{i}}\right) \ddot{\boldsymbol{q}}_{\boldsymbol{i}}+\boldsymbol{C}_{\boldsymbol{i}}\left(\boldsymbol{q}_{\boldsymbol{i}}, \dot{\boldsymbol{q}}_{\boldsymbol{i} \mathbf{1}}\right) \dot{\boldsymbol{q}}_{\boldsymbol{i} \mathbf{2}}=$ $\boldsymbol{Y}_{\boldsymbol{i}}\left(\ddot{\boldsymbol{q}}_{\boldsymbol{i}}, \dot{\boldsymbol{q}}_{\boldsymbol{i} \mathbf{1}}, \dot{\boldsymbol{q}}_{\boldsymbol{i} \mathbf{2}}, \boldsymbol{q}_{\boldsymbol{i}}\right) \boldsymbol{\theta}_{\boldsymbol{i}}$, where $\boldsymbol{Y}_{\boldsymbol{i}} \in \mathcal{R}^{2 \times 3}$ are the regressor, $\boldsymbol{\theta}_{\boldsymbol{i}} \in \mathcal{R}^{3}$ are parameter vector of robots.

The input torque in the experiment is given as

$$
\begin{aligned}
& \tau_{m}=\boldsymbol{Y}_{m}\left(\ddot{\boldsymbol{q}}_{r m}, \dot{\boldsymbol{q}}_{m}, \dot{\boldsymbol{q}}_{r m}, \boldsymbol{q}_{m}\right) \hat{\boldsymbol{\theta}}_{m}-\boldsymbol{K}_{m} \boldsymbol{s}_{m}-\boldsymbol{J}_{m}^{T} \boldsymbol{F}_{o p} \\
& \tau_{s}=\boldsymbol{Y}_{s}\left(\ddot{\boldsymbol{q}}_{r s}, \dot{\boldsymbol{q}}_{s}, \dot{\boldsymbol{q}}_{r s}, \boldsymbol{q}_{s}\right) \hat{\boldsymbol{\theta}}_{\boldsymbol{s}}-\boldsymbol{K}_{\boldsymbol{s}} \boldsymbol{s}_{s}+\boldsymbol{J}_{s}^{T} \boldsymbol{F}_{\text {env }} \\
& \dot{\hat{\boldsymbol{\theta}}}_{\boldsymbol{i}}=-\boldsymbol{\Gamma}_{\boldsymbol{i}}^{-1} \boldsymbol{Y}_{\boldsymbol{i}}\left(\ddot{\boldsymbol{q}}_{\boldsymbol{r} \boldsymbol{i}}, \dot{\boldsymbol{q}}_{\boldsymbol{i}}, \dot{\boldsymbol{q}}_{\boldsymbol{r} \boldsymbol{i}}, \boldsymbol{q}_{\boldsymbol{i}}\right) \boldsymbol{s}_{\boldsymbol{i}}, \\
& \dot{\boldsymbol{q}}_{r i}=\boldsymbol{J}_{\boldsymbol{i}}^{-1}\left\{\dot{\boldsymbol{x}}_{\boldsymbol{i d}}-\boldsymbol{\Lambda}_{\boldsymbol{i}}\left(\boldsymbol{x}_{\boldsymbol{i}}-\boldsymbol{x}_{\boldsymbol{i d}}\right)\right\}, \boldsymbol{s}_{\boldsymbol{i}}=\dot{\boldsymbol{q}}_{\boldsymbol{i}}-\dot{\boldsymbol{q}}_{\boldsymbol{r} i}
\end{aligned}
$$

$\boldsymbol{x}_{\boldsymbol{i}}=\left[x_{i x}, x_{i y}\right]^{T}$ are end-effector position in $x, y$ plane. $\boldsymbol{x}_{\boldsymbol{i d}}, \dot{\boldsymbol{x}}_{\boldsymbol{i d}}, \ddot{\boldsymbol{x}}_{\boldsymbol{i d}}$ are the trajectories of desired linear system. $\ddot{\boldsymbol{x}}_{\boldsymbol{i d}}$ is calculated according to following equation and $\boldsymbol{x}_{\boldsymbol{i d}}, \dot{\boldsymbol{x}}_{\boldsymbol{i d}}$ are calculated by integration of $\ddot{\boldsymbol{x}}_{\boldsymbol{i d}}$

$$
\bar{M}_{m} \ddot{\boldsymbol{x}}_{m d}=\boldsymbol{F}_{m}+\boldsymbol{F}_{\text {op }}, \bar{M}_{s} \ddot{\boldsymbol{x}}_{s d}=\boldsymbol{F}_{s}-\boldsymbol{F}_{\text {env }}
$$

where $\boldsymbol{F}_{\boldsymbol{m}}, \boldsymbol{F}_{\boldsymbol{s}}$ are new input

$$
\begin{aligned}
\boldsymbol{F}_{\boldsymbol{m}}=\left(\boldsymbol{K}_{\boldsymbol{f}}-\boldsymbol{I}\right) \boldsymbol{F}_{\boldsymbol{o p}}-\boldsymbol{K}_{\boldsymbol{f}} \hat{\boldsymbol{F}}_{\boldsymbol{e n \boldsymbol { v }}}\left(t+T_{m}\right)-\boldsymbol{B} \boldsymbol{x}_{\boldsymbol{m} \boldsymbol{d}} \\
\boldsymbol{F}_{\boldsymbol{s}}=\boldsymbol{F}_{\boldsymbol{e n \boldsymbol { v }}}+\boldsymbol{\rho}\left\{\boldsymbol{K}_{\boldsymbol{p}}\left(\boldsymbol{x}_{\boldsymbol{m} \boldsymbol{d}}\left(t-T_{m}\right)-\boldsymbol{x}_{\boldsymbol{s} \boldsymbol{d}}\right)\right. \\
\quad+\boldsymbol{K}_{\boldsymbol{d}}\left(\dot{\boldsymbol{x}}_{\boldsymbol{m} \boldsymbol{d}}\left(t-T_{m}\right)-\dot{\boldsymbol{x}}_{\boldsymbol{s} \boldsymbol{d}}\right) \\
\left.\quad+\boldsymbol{K}_{\boldsymbol{f}}\left(\boldsymbol{F}_{\boldsymbol{o p} \boldsymbol{p}}\left(t-T_{m}\right)-\boldsymbol{F}_{\boldsymbol{e n \boldsymbol { v }}}\right)-\boldsymbol{B} \boldsymbol{x}_{\boldsymbol{s} \boldsymbol{d}}\right\} \\
\hat{\boldsymbol{F}}_{\boldsymbol{e n \boldsymbol { v }}}\left(t+T_{m}\right)=\hat{\boldsymbol{B}}_{\boldsymbol{e n \boldsymbol { v }}}\left(t-T_{s}\right) \dot{\boldsymbol{x}}_{\boldsymbol{m} \boldsymbol{d}} \\
\quad+\hat{\boldsymbol{K}}_{\boldsymbol{e n \boldsymbol { v }}}\left(t-T_{s}\right) \boldsymbol{x}_{\boldsymbol{m} \boldsymbol{d}}+\hat{\boldsymbol{C}}\left(t-T_{s}\right) .
\end{aligned}
$$

The estimation of environment parameter $\hat{\boldsymbol{B}}_{\text {env }}=$ $\operatorname{diag}\left\{\hat{B}_{\text {envx }}, \hat{B}_{\text {envy }}\right\}, \quad \hat{\boldsymbol{K}}_{\text {env }}=\operatorname{diag}\left\{\hat{K}_{\text {envx }}, \hat{K}_{\text {envy }}\right\}, \quad \hat{\boldsymbol{C}}=$ $\operatorname{diag}\left\{\hat{C}_{x}, \hat{C}_{y}\right\}$ are computed according to following equations

$$
\begin{aligned}
\dot{\hat{\boldsymbol{\theta}}}_{\text {envk }} & =-\boldsymbol{P}_{\boldsymbol{k}} \boldsymbol{W}_{\boldsymbol{k}}\left\{\boldsymbol{W}_{\boldsymbol{k}}^{T} \hat{\boldsymbol{\theta}}_{\text {envk }}-F_{\text {envk }}\right\} \\
\frac{d}{d t} \boldsymbol{P}_{\boldsymbol{k}}^{-1} & =\boldsymbol{W}_{\boldsymbol{k}} \boldsymbol{W}_{\boldsymbol{k}}^{T}, \boldsymbol{P}_{\boldsymbol{k}}(0)=p_{0} \boldsymbol{I} \\
\hat{\boldsymbol{\theta}}_{\text {envk }} & =\left[\begin{array}{lll}
\hat{K}_{\text {envk }} & \hat{B}_{\text {envk }} & \hat{C}_{k}
\end{array}\right]^{T} \\
\boldsymbol{W}_{\boldsymbol{k}} & =\left[\begin{array}{lll}
x_{s k} & \dot{x}_{s k} & 1
\end{array}\right]^{T}, \quad(k=x, y)
\end{aligned}
$$

In the above equations, $\bar{M}_{\boldsymbol{i}}, \boldsymbol{K}_{\boldsymbol{i}}, \boldsymbol{\Lambda}_{\boldsymbol{i}}, \boldsymbol{\Gamma}_{\boldsymbol{i}}, \boldsymbol{\rho}, \boldsymbol{K}_{\boldsymbol{f}}, \boldsymbol{K}_{\boldsymbol{p}}, \boldsymbol{K}_{\boldsymbol{d}}$ are design parameter choosen as

$$
\begin{aligned}
& \overline{\boldsymbol{M}}_{\boldsymbol{i}}=\operatorname{diag}\{0.7,0.7\}, \boldsymbol{B}=\operatorname{diag}\{2,2\}, \boldsymbol{K}_{\boldsymbol{i}}=\operatorname{diag}\{10,10\}, \\
& \boldsymbol{\Lambda}_{\boldsymbol{i}}=\operatorname{diag}\{3,3\}, \boldsymbol{\Gamma}_{\boldsymbol{i}}=\operatorname{diag}\{0.1,0.1,0.1\}, \boldsymbol{\rho}=\boldsymbol{I}, p_{0}=100, \\
& \boldsymbol{K}_{\boldsymbol{f}}=\operatorname{diag}\{0.2,0.2\}, \boldsymbol{K}_{\boldsymbol{p}}=\operatorname{diag}\{40,40\}, \boldsymbol{K}_{\boldsymbol{d}}=\operatorname{diag}\{15,15\} .
\end{aligned}
$$

Using this control law, it is guaranteed that $\lim _{t \rightarrow \infty} \boldsymbol{x}_{\boldsymbol{i}}$ $\boldsymbol{x}_{\boldsymbol{i d}}, \dot{\boldsymbol{x}}_{\boldsymbol{i}}-\dot{\boldsymbol{x}}_{\boldsymbol{i d}} \rightarrow 0$ [16], [20], [25]. Thus, the master and slave track the trajectories $\boldsymbol{x}_{\boldsymbol{m} \boldsymbol{d}}, \boldsymbol{x}_{\boldsymbol{s} \boldsymbol{d}}$ which is calculated by (42)(43)(44). Thus, the system behave as linear system. We can analyze stability and performance similarly to section IV even if this control law is used. 